\title{
Academic Psychiatry's Role in Addressing Campus Sexual Assault
}

\author{
Laura Weiss Roberts $^{1} \cdot$ Kendra Dority $^{2} \cdot$ Richard Balon $^{3}$ - Alan K. Louie ${ }^{1}$. \\ Eugene V. Beresin ${ }^{4}$ John H. Coverdale ${ }^{5}$
}

Received: 18 March 2016 / Accepted: 21 March 2016 /Published online: 6 April 2016

(C) Academic Psychiatry 2016

In 2011, the US President, Vice President, and Secretary of Education spotlighted the serious and pervasive issue of sexual assault in institutions of higher learning, offering comprehensive guidance to colleges and universities regarding student rights and needs. In 2014, the White House Task Force to Protect Students from Sexual Assault was established and the "It's On Us" public health campaign was launched, aiming to inspire and engage all members of college campus communities in preventing sexual assault and victimization. At the outset of the campaign, President Obama stated that sexual assault was an "affront to our basic humanity" [1]:

It insults our most basic values as individuals and families, and as a nation. We are a nation that values liberty and equality and justice. And we're a people who believe every child deserves an education ... free from fear of intimidation or violence. And we owe it to our children to live up to those values.

The campaign partnered with student, faculty, and university organizations; the media; the Department of Justice; the

Laura Weiss Roberts

LWRoberts.EIC@gmail.com

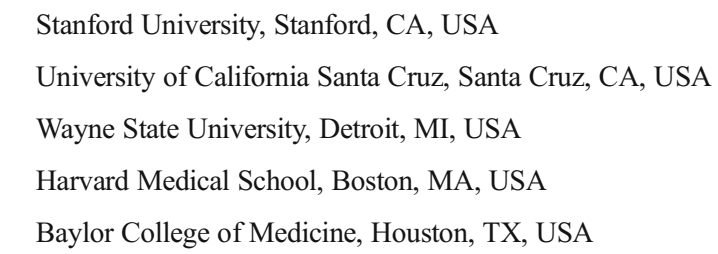

Department of Health and Human Services; and other stakeholders. Through this campaign, efforts were undertaken to reach kindergarteners to high school seniors, first-year university students to college educators, and all levels of leaders in higher education.

Later in 2014, the Massachusetts Institute of Technology (MIT) [2] reported the results of its Community Attitudes on Sexual Assault survey ( $35 \%$ response rate, $N=3844$ participants), endorsing strong, prosocial, respectful, and protective attitudes regarding consensual sexual relations and sexual safety. Nevertheless, $17 \%$ of undergraduate women and $5 \%$ of undergraduate men in this study reported an experience of unwanted sexual behavior during their college careers. When asked about more specific or "labeled" unwanted sexual behaviors, $5 \%$ of the undergraduate women reported being raped, $14 \%$ reported being stalked or followed or receiving repeated unwanted messages that made them feel uncomfortable, and $15 \%$ reported that they had been sexually harassed. The most common perpetrators of these unwanted behaviors and acts of sexual violence were fellow students. These experiences were less commonly reported by undergraduate men and by graduate students in the study. Most survivors of these experiences had talked with someone, such as a friend, family member, or doctor, about what had happened, but only a very small proportion (fewer than $5 \%$ ) formally reported their experience to an institutional or other official. The MIT report emphasized the important positive role of bystanders in shifting social norms, for example, by speaking up against sexist remarks or unwanted behavior, checking in with others about whether they need assistance, and looking out for others by walking them home safely [3]. DeGue [4] has also highlighted the possible benefits of bystander efforts in preventing sexual assault. 
In 2015, the Association of American Universities (AAU) [5] released a report on sexual assault and sexual misconduct at 27 US universities. The survey had a limited (19\%) response rate, with a range of participation from 7 to $53 \%$ at each university and more than 150,000 students participating. The survey instrument was exhaustively comprehensive and highly explicit, covering nonconsensual sexual contact by force or incapacitation, sexual harassment, stalking, and intimate partner violence, among other topics. The analysts acknowledged that non-affected individuals were less likely to participate in the study. They also found that rates of sexual assault are highest among undergraduate women and those identifying as transgender, genderqueer, nonconforming, questioning, or as an identity not listed (TGQN). According to their study, reported rates of nonconsensual sexual contact due to physical force or incapacitation declined over the 4year term of college, with the highest number of incidents occurring in the first years of a student's tenure on campus. The analysts further highlighted that sexual assault remains underreported in institutions of higher learning, while demonstrating that the majority of students who did report an incident had a "very good" or "excellent" experience in the reporting and follow-up process. Groups with the highest rates of sexual victimization (women and TGQN-identified students) were the least likely to respond positively when asked whether a survivor of sexual assault would receive sufficient support and a fair investigation if the incident were officially reported. Finally, the study emphasized the lack of a single "standard" rate of sexual assault or misconduct occurrences at US colleges and universities.

It is disturbing that despite numerous efforts to increase awareness about sexual assault, statistics about sexual violence on college campuses have remained within the same range over decades. In a report that describes the National College Women Victimization (NCWV) study [6] conducted in 1997, Fisher, Cullen, and Turner noted that $2.8 \%$ of female undergraduates experienced either a completed or an attempted rape during the academic year. In the 2015 AAU survey [5], the percentage of undergraduate women reporting "penetration involving incapacitation" was a comparable $2.9 \%$. When the definition of sexual victimization was expanded in the 1997 NCWV study [6] to include any nonconsensual sexual behavior in addition to rape, $15.5 \%$ of college women reported being sexually victimized during the academic year. The 2015 AAU survey [5] found that $26.1 \%$ of female college senior respondents and $29.5 \%$ of TGQN-identified college senior respondents had experienced sexual contact resulting from force or incapacitation during their time on campus. In a study of a sexual assault resistance program targeted towards first-year women at Canadian universities, the 1-year risk of completed rape in a nonintervention (control) group was around $10 \%$ [7].

\section{The Role of Academic Psychiatry}

Academic psychiatrists, among others, are called upon to mobilize their expertise and skills to address critical social issues such as sexual assault, trauma, and emotional health across different developmental life phases, such as when a young person leaves home to attend college. Engaging with colleagues and students to prevent sexual assault, responding to the aftermath of sexual trauma, and fostering policies and practices that enhance safety on college campuses are vital responsibilities that academic psychiatrists should be poised to fulfill. These responsibilities must involve not only providing care to survivors of sexual trauma but also working to shift the perceptions, norms, and general climate relating to sexual assault on campuses. Relevant situational variables include the following: attitudes toward women, sexual minorities, and gender-nonconforming people; gender socialization; rape-supporting beliefs and values; misperceptions and misinterpretations about what constitutes consent; and possibly coercion-supporting peer groups including Greek life and athletics [8]. Alcohol consumption and drug use may also contribute to risk [8-10], and institutional prevention programs and policies can help to reduce such risk.

Academic psychiatrists also may bring important clinical insights in understanding experiential phenomena associated with sexual assault. In responding to incidents of sexual trauma, we must understand the profound and far-reaching effects of sexual assault, which may not be apparent to all of those who are impacted by the experience. Survivors often report feeling numb or detached, and they may feel too vulnerable to provide authentic informed consent for a medical examination. After being sexually assaulted, survivors may experience flashbacks and forms of post-traumatic stress disorder, feel unsettled or impulsive, have difficulty falling or remaining asleep, and/or experience physical symptoms such as headaches or pelvic pain. Students who have been sexually assaulted may have difficulty with their schoolwork and academic performance and in their relationships with intimate partners, friends, teachers, and family members. As with other survivors of sexual assault, students may delay or avoid seeking mental health care, even if they experience significant symptoms of depression, anxiety, post-traumatic stress, or problems associated with alcohol or substance use as a means to cope with victimization. Often, survivors of sexual violence disengage from coursework and eventually leave school due to fear of seeing the perpetrator in class, on campus, or at social gatherings [11] and feelings of shame. These consequences can be attributed to a culture of victim-blaming and lack of awareness among college professors and administrators about the pervasiveness and symptoms of sexual violence [11].

Psychiatrists and other mental health professionals, including clinical psychologists, social workers, and counselors, can play a significant role in assisting student survivors of sexual 
violence. Besides the campus emergency physician, a psychiatrist may be the first professional to whom the sexual assault event is disclosed. Psychiatrists may be well positioned to help the student to process what happened and to inform and empower the student to manage the emotional, psychological, and physical consequences of the experience. Evidencebased treatments for trauma, such as trauma-focused cognitive behavioral therapy and supportive group therapy, may be helpful in this process. A psychiatrist may also provide support when a student reports the assault to campus officials, which triggers investigative and judicial procedures that can be emotionally difficult and feel "re-victimizing." When a student who has been sexually assaulted has pre-existing mental health issues, a psychiatrist can provide appropriate clinical care during the period of transition and recovery after the assault experience.

Psychiatrists may also assist other individuals affected by sexual assault - and many are affected, including perpetrators of sexual assault (who themselves have sometimes been similarly assaulted), individuals accused of perpetrating sexual assault, bystanders and others who feel a sense of responsibility for the assault, friends, loved ones, and family members. Psychiatrists may also be of help when seeking to clarify impulsive behavior as opposed to systematic, purposeful behavior of a sexual predator on campus. In addition, faculty and administrators who conduct investigations and establish policy are also affected by the event, particularly when they have little formal preparation for dealing with serious trauma. Psychiatrists may provide direct care, offer consultation, and help create trauma-informed strategies for managing these complex issues.

Despite the care and assistance they can provide, psychiatrists have heretofore had a relatively limited role in addressing sexual violence on college campuses. This limited role is due in part to the overall crisis in college mental health, that is, the lack of adequate systems of care within US colleges and universities-including the absence of psychiatry services on many, especially smaller, campuses - in spite of the pervasive mental health issues that college students currently face [12]. When they are involved, psychiatrists primarily help the survivors of sexual violence with the aftermath of such an experience, yet the most important factor in a holistic social response to the epidemics of sexual violence on college campuses lies in primary prevention. Various preventative measures and programs have been implemented and tested (e.g., bystander intervention [13, 14], theater of the oppressed [11], women's self-defense training $[15,16]$, rape prevention education). However, the evidence for the efficacy of these programs is mixed at best [4], and the rates of sexual violence on college campuses remain high. One randomized controlled trial of a sexual assault resistance program involving first-year female students at three universities in
Canada, however, significantly reduced the occurrence of rape and attempted rape in the intervention compared to a control group [7].

The current state of affairs is wholly unacceptable and intolerable; one rape or sexual victimization incident, certainly, is one too many and has damaging, life-changing effects. Academic psychiatry must become more involved in finding long-term solutions to ending sexual assault, by both assisting survivors of sexual violence and promoting primary prevention. In order to work toward the eradication of sexual violence on college and university campuses, we propose several measures.

\section{Increased Involvement in College Mental Health}

Academic psychiatry must increase its involvement in providing mental health services to college students. These services include specialized treatment for survivors of sexual violence as well as prevention-based methods that aim to shift campus climates and current social attitudes about sexual assault. Specialized centers for assisting survivors of sexual violence should be established on college campuses. There is currently a profound shortage of certified mental health professionals on college campuses and almost no staff psychiatrists. In the 2012 report of the Association for University and College Counseling Center Directors [17], the average student to paid professional mental health staff was 1:664 for small colleges, 1:1864 for mid-sized colleges, and 1:2731 for large universities. In the same report, the average percent of students seeking counseling services was $9-12 \%$ at small colleges and 6-7 \% at large universities [17]. Not only is there a lack of input from psychiatry but there is clearly a failure to provide adequate personnel to assist students in need. We need to increase the trained workforce and expand services for college students as well as modify the barriers that prevent students from seeking help, particularly in areas such as sexual assault that are so highly sensitive and stigmatized.

One of the reasons why psychiatry does not have greater involvement in addressing sexual health and sexual violence may be prejudicial views toward the field of psychiatry. These attitudes may be changing, but it is important to emphasize that an individual who has survived sexual assault should not be labeled as mentally ill — survivors are living out predictable sequela of an undeserved and tragic human experience. In this context, psychiatrists along with other specialized physicians and health professionals have knowledge and skill that will help survivors and other impacted parties, who do deserve every possible resource to assist them as they move through and beyond this terrible period in their lives, changed but, with hope, with better outcomes. 
In order to break the culture of silence around sexual assault and to empower students to challenge social norms, these centers should include "safe spaces" and "safe conversations" where students of all gender identities can not only discuss the trauma of sexual violence but also challenge gendered discrimination and gender role socialization [18]. Recent studies have shown that sexual violence, assault, and harassment have been disproportionately reported by women and TGQNidentified students; thus, mental health providers on college campuses must be trained to address the needs of these specific student groups through affirmative, supportive care [19].

\section{Expanded Education in Sexual Health}

Education in sexual health, including sexual violence prevention programs, should be expanded to reach all college and university campuses. Students should be involved in designing and delivering curricula for these programs to cultivate a supportive, respectful, and consent-based culture from within their peer groups [20]. Sexual health education programs should be "student-facing" and inclusive of students of all gender identities, because sexual assault affects people of all genders and all students must be involved in shifting perceptions about sexual assault and gendered forms of socialization [21]. These programs should aim to promote a gender egalitarian, non-heterosexist, trans-affirmative campus climate in which sexually marginalized and gender-nonconforming students can feel safe [22].

\section{Additional Training for Psychiatrists}

Psychiatrists, and especially psychiatry residents, should be trained to help survivors of sexual violence on campuses by counseling and supporting students and by providing appropriate guidance and treatment. This training should include cultivating a sensitive, empathic approach to discussing and treating sexual trauma with survivors [23]. Psychiatry residency programs should embrace an inclusive education in which gender-related issues, especially those pertinent to women and to sexually marginalized and gender-nonconforming individuals, are incorporated into the curriculum and in clinical training opportunities [24].

\section{Additional Training for Campus Leaders}

Academic psychiatrists should assist in developing campuswide training programs aimed at educating campus leaders, such as college officials, administrators, professors, counselors, and resident advisors, in how to appropriately and effectively respond to incidents of sexual violence in ways that support survivors [25]. Given the low rates by which students report sexual assault to official campus agencies [5] and the higher likelihood that they will discuss the incident with a peer [25], supervised peer counseling services should be established to provide safe spaces where students can discuss their experience in confidence and learn about further resources available to them on campus. Moreover, medical and mental health professionals working on college campuses should be informed about the situational factors that may contribute to risk and should be trained to sensitively, validly, and routinely screen for a history of sexual assault. Potential barriers to screening, including prejudicial or false beliefs about sexual assault, survivors, and perpetrators, should be addressed. One related study of medical students indicated that sexual assault education can diminish prejudicial or false attitudes about rape and promote screening for sexual assault [26].

\section{Assessments of Prevention Programs}

Academic psychiatry should be involved in assessing the efficacy of various prevention programs that are already in place on college campuses, including bystander intervention and sexual assault resistance programs. Prevention should be informed by routine screening for sexual assault and by measures that identify high alcohol consumption and substance use as well as other situational factors that may contribute to risk. On the basis of these assessments, academic psychiatrists should also be involved in inventing and evaluating new programs that have lasting effects in the campus community by shifting both attitudinal and behavioral norms and that are informed by specific institutional contexts and community needs [20].

As these suggestions demonstrate, academic psychiatrists should advocate for an increased presence on college campuses and for an increased role in reducing college campus sexual assaults. We must be attentive to the specific needs of populations that have historically experienced higher rates of sexual violence-women and TGQN-identified studentswhile also working to support, educate, and strengthen the policy approaches of entire campus communities. In working towards ending sexual assault on our campuses, we must become informed about the evidence on the efficacy of intervention programs and enact holistic solutions that aim not only to better assist survivors but also to prevent future acts of sexual violence and, ultimately, to shift how we talk about, respond to, and perceive sexual violence.

\section{Compliance with Ethical Standards}

Disclosure On behalf of all authors, the corresponding author states that there is no conflict of interest. 


\section{References}

1. The White House Office of the Press Secretary. Remarks by the President at "It's On Us" campaign rollout. Available at https:// www.whitehouse.gov/the-press-office/2014/09/19/remarkspresident-its-us-campaign-rollout. Accessed 2 February 2016.

2. Massachusetts Institute of Technology. Survey results: 2014 community attitudes on sexual assault. October 27, 2014. Available at http://web.mit.edu/surveys/health/MIT-CASA-Survey-Summary. pdf. Accessed 2 February 2016

3. Massachusetts Institute of Technology. MIT Health \& Wellness Surveys: 2014 community attitudes on sexual assault. Survey update from the Chancellor, April 2015. Available at http://web.mit. edu/surveys/health/slides/. Accessed 2 February 2016.

4. DeGue S. Preventing sexual violence on college campuses: lessons from research and practice. Prepared for the White House Task Force to Protect Students from Sexual Assault. April 2014. Available at http://notalone.gov/assets/evidence-based-strategies-for-theprevention-of-sv-perpetration.pdf. Accessed 2 February 2016.

5. Cantor D, Fisher B, Chibnall S, Townsend R, Lee H, Bruce C, et al. Report on the AAU campus climate survey on sexual assault and sexual misconduct. Association of American Universities. September 21, 2015. Available at: https://www.aau.edu/ClimateSurvey. Accessed 2 February 2016.

6. Fisher BS, Cullen FT, Turner MG. The sexual victimization of college women. National Institute of Justice. 2000. Report No. NCJ-182369.

7. Senn CY, Eliasziw M, Barata PC, Thurston WE, et al. Efficacy of a sexual assault resistance program for university women. NEJM. 2015;372:2326-35.

8. Adams-Curtis LE, Forbes GB. College women's experiences of sexual coercion. Trauma Violence Abuse. 2004;5:91-122.

9. Testa M, Livingston JA. Alcohol consumption and women's vulnerability to sexual victimization: can reducing women's drinking prevent rape? Subst Use Misuse. 2009;44:1349-76.

10. Zinzow HM, Thompson M. A longitudinal study of risk factors for repeated sexual coercion and assault in U.S. College men. Arch Sex Behav. 2015;44:213-22.

11. Christensen MC. Using theater of the oppressed to prevent sexual violence on college campuses. Trauma Violence Abuse. 2013;14: 282-94.

12. Balon R, Beresin EV, Coverdale JH, Louie AK, Roberts LW. College mental health: a vulnerable population in an environment with systemic deficiencies. Acad Psychiatry. 2015;39:495-7.

13. Katz J, Moore J. Bystander education training for campus sexual assault prevention: an initial meta-analysis. Violence Vict. 2013;28: 1054-67.
14. Alegria-Flores K, Raker K, Pleasants RK, Weaver MA, Weinberger $M$. Preventing interpersonal violence on college campuses: the effect of one act training on bystander intervention. J Interpers Violence. 2015.

15. Gidycz CA, Dardis CM. Feminist's self-defense and resistance training for college students: a critical review and recommendations for the future. Trauma Violence Abuse. 2014;15:32233.

16. McCaughey M, Cermele J. Changing the hidden curriculum of campus rape prevention and education: women's self-defense as a key protective factor for a public health model of prevention. Trauma Violence Abuse. 2015.

17. Reetz DR, Barr V, Krylowicz B. The Association for University and College Counseling Center Directors annual survey. Reporting period: September 1, 2012 through August 31, 2013. Available at http://files.cmeglobal.com/AUCCCD Monograph Public 2013. pdf. Accessed 8 February 2016.

18. McCauley HL, Casler AW. College sexual assault: a call for trauma-informed prevention. J Adolesc Health. 2015;56:584-5.

19. American Psychological Association. Guidelines for psychological practice with transgender and gender nonconforming people. Am Psychol. 2015;70(9):832-64.

20. Edwards KM, Jones LM, Mitchell KJ, Hagler MA, Roberts LT. Building on youth's strengths: a call to include adolescents in developing, implementing, and evaluating violence prevention programs. Psychol Violence. 2016;6:15-21.

21. Stewart AL. The men's project: a sexual assault prevention program targeting college men. Psychol Men Masc. 2014;15:481-5.

22. Woodford MR, Kulick A, Atteberry B. Protective factors, campus climate, and health outcomes among sexual minority students. J Divers High Educ. 2015;8:73-87.

23. Derenne J, Roberts LW. Considering the effects of sexual trauma when teaching physicians about human sexuality. Acad Psychiatry. 2010;34:409-13.

24. Gold LH, Epstein SA. Formal training in women's issues in psychiatry: a survey of psychiatry residency training directors. Acad Psychiatry. 2006;30:403-9.

25. Orchowski LM, Gidycz CA. Psychological consequences associated with positive and negative responses to disclosure of sexual assault among college women: a prospective survey. Violence Against Women. 2015;21:803-23.

26. Milone JM, Burg MA, Duerson MC, Hagen MG, Pauly RR. The effect of lecture and a standardized patient encounter on medical student rape myth acceptance and attitudes toward screening patients for a history of sexual assault. Teach Learn Med. 2010;22:37-44. 